\section{Evaluación de la actividad cerebro-cerebelar cruzada para determinar la lateralidad del lenguaje en pacientes con tumores cerebrales}

\author{
CAROLINA MÉNDEZ-ORELLANA ${ }^{1, \mathrm{a}, \mathrm{e}, \text {, }}$ \\ LEONARDO ARRAÑO-CARRASCO ${ }^{2}$, \\ BÁRBARA CORTÉS-RIVERA ${ }^{3, b, e}$, KARINA SANDOVAL-LEÓN ${ }^{4,9, c, e,}$, \\ JOSÉ LORENZONI-SANTOS ${ }^{5}$, PABLO VILLANUEVA-GARÍN ${ }^{5}$, \\ RICARDO ROJAS-VALDIVIA ${ }^{5}$, TEOBALDO MÉNDEZ-ORTEGA ${ }^{6}$, \\ JAIME MONSALVE-ROSALES ${ }^{6}$, PAULO FLORES-KRUUSE ${ }^{6,7}$, \\ PABLO CARMONA-RAMMSY, DAVID ROJAS-PINTO ${ }^{9}$, \\ ÁLVARO REYES-PONCE ${ }^{10, \mathrm{d,f}, \text { FRANCISCO MERY-MUÑOZ }}{ }^{5 *}$
}

\section{Evaluation of the crossed cerebro-cerebellar activation to determine language lateralization in patients with brain tumors}

Background: The crossed cerebro-cerebellar (CCC) activation facilitates the diagnosis of cortical language lateralization, but needs to be explored with language tasks suitable for patients with different age ranges, educational attainment and eventual presence of language deficits. Aim: To determine the effect of demographic variables in the performance of three language tasks in healthy volunteers and to determine the CCC activation of these tasks as a functional magnetic resonance imaging (fMRI) paradigm in brain tumor patients. Material and Methods: The behavioral performance (correct responses and reaction time) of three language tasks (verbal fluency, semantic and phonological decision tasks) was first examined in 76 healthy volunteers balanced by age and educational level. Later, these tasks were implemented as fMRI paradigms to explore CCC language activation of 20 patients with potential diagnosis of brain tumors. Results: The performance of the verbal fluency task was affected by age. The CCC language activation was reproducible with the semantic and phonological tasks. The combination of the tasks determined typical and atypical language lateralization in $60 \%$ and $40 \%$ of our patients, respectively. Conclusions: The verbal fluency task must be implemented with care as a clinical fMRI paradigm. Our results suggest that semantic and phonological tasks can be a good alternative for brain tumor patients with language deficits.

(Rev Med Chile 2021; 149: 689-697)

Key words: Brain Neoplasms; Cerebellum; Functional Laterality; Language; Magnetic Resonance Imaging.
${ }^{1}$ Carrera de Fonoaudiología, Facultad de Medicina, Universidad Católica de Chile. Santiago, Chile.

${ }^{2}$ Departamento de Radiología, Facultad de Medicina, Universidad Católica de Chile. Santiago, Chile.

3Escuela de Fonoaudiología, Facultad de Salud, Universidad Santo Tomás. Santiago, Chile.

${ }^{4}$ Escuela de Fonoaudiología, Facultad de Salud, Universidad Católica Silva Henríquez. Santiago, Chile.

${ }^{5}$ Departamento de Neurocirugía, Facultad de Medicina, Universidad Católica de Chile. Santiago, Chile. ${ }^{6}$ Servicio de Neurocirugía, Complejo Asistencial Doctor Sótero del Río. Santiago, Chile.

${ }^{7}$ Servicio de Neurocirugía, Hospital del Trabajador. Santiago, Chile.

${ }^{8}$ Servicio de Neurocirugía, Hospital Base de Puerto Montt. Puerto Montt, Chile. ${ }^{9}$ Servicio de Neurocirugía, Hospital Barros Luco Trudeau. Santiago, Chile. ${ }^{10}$ Escuela de Kinesiología, Facultad de Ciencias de la Rehabilitación. Universidad Andrés Bello. Viña del Mar, Chile.

Doctor en Neurociencias, Erasmus MCUniversity Medical Center Rotterdam, Países Bajos.

'Magister en Audiología, Universidad Andrés Bello, Santiago, Chile. "Magíster en Trastornos del Lenguaje, Universidad de Valparaíso. Valparaíso, Chile.

dDoctor en Ciencias Médicas, Edith Cowan University, Perth, Australia. eFonoaudióloga.

fKinesiólogo.

Trabajo financiado por proyecto FONDECYT de inicio 11150429.

Los autores declaran no tener conflictos de interés.

Recibido el 6 de julio de 2020, aceptado el 16 de marzo de 2021.

Correspondencia a:

Francisco Mery Muñoz, MD Departamento de Neurocirugía, Facultad de Medicina, Universidad Católica de Chile.

franciscomery@neurocirugiauc.cl

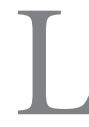

a resonancia magnética funcional (RMf) es una técnica incorporada a la práctica clínica que permite establecer la relación de lesiones encefálicas con áreas elocuentes ${ }^{1}$. Al tratarse de una técnica no invasiva e informar so- bre la relación espacial entre las áreas del lenguaje y la lesión cerebral $^{2}$, se ha posicionado como técnica de elección en el estudio y planificación terapéutica de lesiones encefálicas.

La RMf presenta algunos desafíos para ser un 
método confiable en la práctica clínica. Uno de estos es el efecto de la lesión en la señal hemodinámica BOLD (Blood-Oxygen-Level-Dependent-imaging) en áreas elocuentes ${ }^{3,4}$. La activación cerebro-cerebelar cruzada (CCC) es un patrón de activación reportado en tareas de fluidez verbal (FV) en voluntarios sanos y pacientes con tumores cerebrales ${ }^{5,6}$. Esta activación es considerada como una herramienta diagnóstica que contribuye a determinar la lateralización del lenguaje, enfocándose en la activación cerebelar contralateral a la activación supratentorial, cuya señal BOLD no se ve interferida por la lesión tumoral ${ }^{7}$.

Otro importante desafío es la selección de la tarea lingüística para ser realizada dentro del resonador, la ejecución de esta debe activar regiones específicas del proceso lingüístico, sin interferencia de otros procesos cognitivos ${ }^{8}$. Por otra parte, esta tarea debe ser acorde a las características demográficas y capacidades cognitivo-lingüísticas del paciente ${ }^{9-12}$. Considerando estos factores, se asegura que la activación cerebral corresponda al fenómeno lingüístico en estudio. La actual recomendación para evaluar el procesamiento del lenguaje es la combinación de múltiples tareas para lograr activar el circuito responsable de su procesamiento ${ }^{13}$, siendo la tarea de FV la más utilizada en estudios clínicos. Estudios han propuesto otras tareas, tales como la generación de palabras, generación de oraciones ${ }^{14,15}$ y escucha pasiva. Sin embargo, son tareas de mayor complejidad lingüística (en especial aquellas enfocadas a nivel de expresión del lenguaje) difíciles de implementar en pacientes adultos mayores y pacientes con alteraciones de lenguaje.

En Chile, la RMf se está instaurando como examen rutinario prequirúrgico para evaluar la funcionalidad de áreas elocuentes. Dependiendo del centro médico, se realizan diversas tareas para evaluar la lateralización cerebral del lenguaje y, por lo tanto, no existen tareas que hayan tenido un proceso de adaptación cultural y lingüística previo a su implementación en pacientes con alteraciones del lenguaje. En consecuencia, los objetivos del presente estudio son: 1) evaluar las variables de edad y nivel educacional en el rendimiento de tareas de RMf de lenguaje en voluntarios sanos, optimizando su diseño previo a su implementación clínica y 2) evaluar la concordancia de las tareas de lenguaje para determinar el patrón de activación CCC del lenguaje en personas adultas con tumores cerebrales.

\section{Materiales y Métodos}

\section{Participantes}

En el pilotaje conductual de los estímulos de las tareas RMf participó un grupo de 76 voluntarios sanos (edad promedio 56,07 años, $\mathrm{DE}=19,90 ; 13$ zurdos) balanceado por sexo y nivel educacional. Los criterios de inclusión fueron ser mayor de 18 años, sin antecedentes de alteraciones neurológicas o psiquiátricas. En caso de los mayores de 60 años, presentar puntaje $\geq 21$ en test Mini-Mental, según la validación chilena ${ }^{16}$. Los criterios de exclusión fueron presentar alteraciones auditivas y antecedentes de alteraciones del habla o lenguaje.

En el pilotaje de la actividad CCC de estas tareas de lenguaje RMf se reclutaron 20 personas con sospecha de tumor cerebral (edad promedio 42,15 años, $\mathrm{DE}=14,98 ; 11$ mujeres; 4 con escolaridad menor a 12 años). En Tabla 1 se presentan datos demográficos de los participantes y resultados de estudio patológico de los tumores cerebrales. Los criterios de inclusión para las personas con sospecha de tumor cerebral fueron: ser mayores de 18 años y tener diagnóstico de trabajo de probable tumor cerebral intraaxial e indicación quirúrgica consecuente (basado en resonancia magnética [RM] anatómica preoperatoria y cuadro clínico). Los criterios de exclusión fueron no estar en condiciones para dar consentimiento informado $\mathrm{y}$ tener contraindicaciones para ser sometidos a una sesión de RMf.

Este estudio contó con la aprobación de Comité Ético Científico de las instituciones asociadas al proyecto, Pontificia Universidad Católica de Chile (ID15-302), Servicio Metropolitano de Salud Sur Oriente y Servicio de Salud Metropolitano Sur.

\section{Diseño de tareas de RMf de lenguaje}

Se diseñaron 3 tareas validadas en contexto clínicos para evaluar fluidez verbal (FV $)^{17,18}$, procesamiento semántico (SEM) y procesamiento fonológico $(\mathrm{FON})^{19,20}$. Los estímulos de las tareas fueron seleccionados por dos fonoaudiólogas en base a frecuencia, metría (palabras bisilábicas) e imaginabilidad. Las tareas fueron grabadas manteniendo una prosodia natural. Las tareas siguieron un diseño en bloques interponiendo 
Tabla 1. Caracterización demográfica de voluntarios sanos y pacientes

\begin{tabular}{|c|c|c|}
\hline & $\begin{array}{l}\text { Voluntarios sanos } \\
\qquad(n=76)\end{array}$ & $\begin{array}{l}\text { Pacientes } \\
(n=20)\end{array}$ \\
\hline Edad en años, media (DE) [rango] & $56,08(19,90)[20-84]$ & $42,15(14,98)[20-70]$ \\
\hline $\begin{array}{l}\text { Sexo, n (\%) } \\
\text { Femenino } \\
\text { Masculino }\end{array}$ & $\begin{array}{l}38(50) \\
38(50)\end{array}$ & $\begin{array}{l}11(55) \\
9(45)\end{array}$ \\
\hline $\begin{array}{l}\text { Lateralidad dominante (EHI), n (\%) } \\
\text { Diestro } \\
\text { Zurdo }\end{array}$ & $\begin{array}{l}63(82,9) \\
13(17,1)\end{array}$ & $20(100)$ \\
\hline $\begin{array}{l}\text { Nivel educacional, n (\%) } \\
\text { Menor igual a } 12 \text { años (baja escolaridad) } \\
\text { Más de } 12 \text { años }\end{array}$ & $\begin{array}{l}38(50) \\
38(50)\end{array}$ & $\begin{array}{l}12(60) \\
8(40)\end{array}$ \\
\hline $\begin{array}{l}\text { Histopatología del tumor, n } \\
\text { Astrocitoma difuso } \\
\text { Astrocitoma policítico } \\
\text { Astrocitoma anaplásico } \\
\text { Oligodendroglioma } \\
\text { Glioblastoma multiforme } \\
\text { Tuberculoma } \\
\text { Displasia cortical } \\
\text { Metástasis } \\
\text { No concluyente }\end{array}$ & & $\begin{array}{l}7 \\
1 \\
1 \\
2 \\
5 \\
1 \\
1 \\
1 \\
1\end{array}$ \\
\hline $\begin{array}{l}\text { Lateralidad del tumor, } \mathrm{n} \\
\text { Hemisferio izquierdo } \\
\text { Hemisferio derecho }\end{array}$ & & $\begin{array}{c}12 \\
8\end{array}$ \\
\hline $\begin{array}{l}\text { Localization del tumor, n } \\
\text { Frontal } \\
\text { Temporal } \\
\text { Parietal } \\
\text { Fronto-temporal } \\
\text { Fronto-parietal } \\
\text { Fronto-parieto-insular }\end{array}$ & & $\begin{array}{l}6 \\
4 \\
2 \\
4 \\
3 \\
1\end{array}$ \\
\hline
\end{tabular}

$\mathrm{DE}=$ Desviación estándar; EHI=Edinburgh Handedness Inventory.

una condición control a una experimental. Diseño y características de las tareas se presentan en Tabla 2.

Las tareas fueron programadas y sincronizadas utilizando software Presentation v13.0 (Neurobehavioral Systems Inc., Albany, CA, EE.UU.). Los voluntarios sanos ejecutaron las tareas en un computador portátil y se obtuvo registro de respuestas correctas (RC) y tiempo de reacción (TR) para cada una de las tareas. Los pacientes ejecutaron las 3 tareas dentro de un resonador 3T Ingenia Gyroscan (Philips, Best, Países Bajos). Los estímulos se presentaron de forma biaural mediante audífonos compatibles con RM (MR Confon, Magdeburg, Alemania). El rendimiento (RC y TR) de las tareas SEM y FON se registraron mediante una botonera de fibra óptica diseñada por Cambridge Research Systems (Rochester, Reino Unido). En caso de la tarea FV, se solicitó pensar la respuesta, evitando gesticular o verbalizar la palabra en voz alta (respuesta no verbal, modalidad cubierta). Dentro de un rango de 1 a 3 días previo a la sesión de RMf, los pacientes practicaron la tarea fuera del resonador, con el fin de verificar su rendimiento previo a la sesión de RMf (sesión de entrenamiento). 
Tabla 2. Caracterización demográfica de voluntarios sanos y pacientes

\begin{tabular}{|c|c|c|c|}
\hline Tareas & Fluidez verbal (FV) & Decisión semántica (SEM) & Decisión fonológica (FON) \\
\hline Diseño & $\begin{array}{l}5 \text { bloques de condición control } \\
5 \text { bloques de condición } \\
\text { experimental }\end{array}$ & $\begin{array}{l}6 \text { bloques de condición control } \\
6 \text { bloques de condición } \\
\text { experimental }\end{array}$ & $\begin{array}{l}6 \text { bloques de condición control } \\
6 \text { bloques de condición } \\
\text { experimental }\end{array}$ \\
\hline Duración & Bloques de 30 segundos & Bloques de 21 segundos & Bloques de 21 segundos \\
\hline Estímulos & $\begin{array}{l}\text { Condición control: tono agudo } \\
(2000 \mathrm{~Hz}) \text { y un tono grave } \\
(400 \mathrm{~Hz}) \\
\text { Condición experimental: se } \\
\text { presentaron sustantivos, para los } \\
\text { cuales los participantes tenían que } \\
\text { pensar en una acción (verbo) }\end{array}$ & $\begin{array}{l}\text { Condición control: ruido café } \\
(8 \mathrm{~Hz}) \text { y un tono grave }(400 \mathrm{~Hz}) \text {. } \\
\text { Participantes debían indicar } \\
\text { cuando escuchaban el ruido } \\
\text { Condición experimental: se } \\
\text { presentaron pares de sustantivos } \\
\text { y se solicitó indicar cuando ambos } \\
\text { se asociaban semánticamente }\end{array}$ & $\begin{array}{l}\text { Condición control: ruido café } \\
(8 \mathrm{~Hz}) \text { y un tono grave }(400 \mathrm{~Hz}) \text {. } \\
\text { Participantes debían indicar } \\
\text { cuando escuchaban el ruido } \\
\text { Condición experimental: se } \\
\text { presentaron pares de sustantivos } \\
\text { y se solicitó indicar cuando ambos } \\
\text { rimaban }\end{array}$ \\
\hline
\end{tabular}

\section{Diseño y análisis de tareas de $R M f$}

Los parámetros de adquisición de imágenes estructurales secuencia gradiente ponderada en T1 volumétrica fueron de tamaño $1 \mathrm{~mm}$ voxel isotrópico, FOV $240 \times 240 \mathrm{~mm}(\mathrm{TR}=8,2 \mathrm{~ms}$ $\mathrm{TE}=3,2 \mathrm{~ms}$ ). Los parámetros funcionales se diseñaron en base a los tiempos de reacción del grupo piloto de voluntarios sanos, siendo una secuencia $\mathrm{T}^{*}$ con $\mathrm{TE}=30 \mathrm{~ms}$ FOV $230 \times 230 \mathrm{~mm}, 3 \mathrm{~mm}$ voxel isotrópico y $\mathrm{TR}=3000 \mathrm{~ms}$ para tarea $\mathrm{FV}$ y TR $=3500 \mathrm{~ms}$ para las tareas SEM y FON. Las imágenes de RMf fueron analizadas usando SPM8 (Statistical Parametrical Mapping, London, UK). Inicialmente fueron manualmente alineadas a la comisura anterior, luego fueron corregidas temporalmente, realineadas, corregistradas con las imágenes anatómicas y suavizadas mediante un kernel gaussiano de $6 \mathrm{~mm}$ (FWHM). Siguiendo el protocolo de análisis prequirúrgico, los datos no fueron normalizados sino reformateados preservando el tamaño del voxel ${ }^{13}$. El análisis estadístico de las imágenes funcionales consistió en utilizar pruebas del tipo Student t-test con p de 0,05 mediante la opción family wise error de SPM8, lo que implica $5 \%$ de probabilidad de obtener falsos positivos. Se controló que las adquisiciones no presentaran movimientos superiores a las dimensiones de voxel de adquisición y que presentaran patrones de activación coherente. Las lesiones y los patrones de actividad CCC fueron revisadas e informadas por un neuroradiólogo experto uti- lizando el software MRIcron (https://www.nitrc. org/projects/mricron) siguiendo el protocolo de Méndez-Orellana et $\mathrm{al}^{6}$.

\section{Análisis de datos}

Los datos continuos se describieron utilizando media y desviación estándar (DE). Los datos nominales se describieron utilizando porcentajes. En los voluntarios sanos, diferencias en las RC para las tareas se analizaron bajo un modelo de regresión lineal múltiple ajustado por edad, sexo y nivel educacional (dicotomizado en mayor o menor igual a 12 años de escolaridad). En los pacientes, la concordancia de la activación cortical entre las tareas para determinar la lateralidad del lenguaje se analizó mediante índice Kappa. Se utilizó programa IBM SPSS v20.0 para análisis estadístico y se consideró un nivel de significancia de 5\%.

\section{Resultados}

Resultados del pilotaje conductual de los estímulos de RMf en voluntarios sanos

En las tareas SEM, FON y VF los participantes sanos lograron un porcentaje de RC de 98,6\%, $97,8 \%$ y $90,79 \%$, respectivamente. El promedio de TR para la tarea FV fue de 2,42s ( $\mathrm{DE}=0,08)$, para SEM fue de $3,13 \mathrm{~s}(\mathrm{DE}=0,05)$ y para FON de $3,17 \mathrm{~s}(\mathrm{DE}=0,04)$.

El resultado del análisis de regresión múltiple para determinar la asociación de las variables sexo, 
edad y nivel educacional considerando el número de RC en las tareas demostró que solo para la tarea de FV existió asociación con la edad, a mayor edad peor rendimiento (Tabla 3 ).

\section{Rendimiento de los pacientes en sesión de entrenamiento previo y durante $R M f$}

De los 20 pacientes incluidos, solo 1 paciente (no 21) no logró obtener un puntaje mínimo de corte en la sesión de entrenamiento previo a la RMf para las tareas FV y FON, dado su cuadro de afasia grave. El rendimiento de los pacientes en la sesión de entrenamiento para las tareas SEM, FON y VF fue de $96,9 \%, 96,3 \%$ y $86 \%$, respectivamente. El promedio de TR para la tarea FV fue de 2,71s $(\mathrm{DE}=0,09)$, para SEM fue de $3,18 \mathrm{~s}(\mathrm{DE}=0,06)$ y para FON de 3,20s ( $\mathrm{DE}=0,05)$.

El rendimiento de los pacientes durante RMf en las tareas SEM, FON fue de 95,7\% y $94,9 \%$. El promedio de TR para la tarea SEM fue de $3,23 \mathrm{~s}$ $(\mathrm{DE}=0,06)$ y para FON de $3,31 \mathrm{~s}(\mathrm{DE}=0,08)$. Dado a que las respuestas de la tarea FV no son verbalizadas, no se obtienen registros de $\mathrm{RC}$ y $\mathrm{TR}$ dentro del resonador.

\section{Lateralización del lenguaje en pacientes con tumor cerebral}

Las tres tareas coincidieron en determinar la actividad CCC en 9 pacientes (45\%), 7 con lateralidad típica de lenguaje y 2 con lateralidad atípica. De forma independiente, la tarea FV determinó CCC en $70 \%$ de los pacientes (14/20), la tarea SEM en $85 \%$ de los pacientes $(17 / 20)$ y la tarea FON en 85\% (17/20) de los pacientes. Solo 2 pacientes mostraron actividad ipsilateral en el cerebro y cerebelo. Los resultados de activación cerebro-cerebelar cruzada y patrón de activación de lateralidad de lenguaje según patrón de activación de las tareas de lenguaje se reportan en la Tabla 4.

El análisis de concordancia entre las tareas mostró 73,68\% de acuerdo entre las tareas FV y SEM (Kappa $=0,27 ; \mathrm{p}=0,11), 73,68 \%$ de acuerdo entre las tareas FV y FON (Kappa $=0,13$; $\mathrm{p}=0,28)$ y $78,95 \%$ de acuerdo entre las tareas SEM y FON (Kappa $=0,38 ; p=0,04)$. La Figura 1 ilustra un caso de concordancia de estas tareas en un paciente.

\section{Discusión}

El presente estudio evaluó el rendimiento de 3 tareas de RMf de lenguaje en voluntarios sanos con alta (con más de 12 años de educación) y baja escolaridad (menor o igual a 12 años de educación). En base a los resultados, se exploró el tiempo de presentación de estímulo y el tiempo de respuesta óptimo para implementar estas tareas como paradigmas de RMf en 20 personas adultas con

Tabla 3. Análisis de regresión múltiple para determinar asociación de las variables sexo, edad y nivel educacional en el rendimiento de las tareas de lenguaje en voluntarios sanos

\begin{tabular}{|llccccc|}
\hline Tarea & Variable & Coeficiente & EE & Valor $\mathbf{p}$ & Límite inferior & Límite superior \\
FV & Sexo & 0,231 & 0,339 & 0,499 & $-0,446$ & 0,908 \\
& Escolaridad & $-0,331$ & 0,339 & 0,333 & $-1,008$ & 0,346 \\
& Edad & $-0,113$ & 0,009 & 0,000 & $-0,130$ & $-0,096$ \\
& cons & 48,314 & 0,566 & 0,000 & 47,186 & 49,441 \\
SEM & Sexo & $-0,027$ & 0,106 & 0,803 & $-0,237$ & 0,184 \\
& Escolaridad & $-0,081$ & 0,106 & 0,449 & $-0,291$ & 0,130 \\
& Edad & $-0,005$ & 0,003 & 0,097 & $-0,010$ & 0,001 \\
& cons & 36,003 & 0,176 & 0,000 & 35,652 & 36,355 \\
\multirow{2}{*}{ FON } & Sexo & $-0,211$ & 0,114 & 0,069 & $-0,438$ & 0,017 \\
& Escolaridad & $-0,054$ & 0,114 & 0,640 & $-0,281$ & 0,174 \\
& Edad & $-0,003$ & 0,003 & 0,352 & $-0,008$ & 0,003 \\
& cons & 35,758 & 0,190 & 0,000 & 35,378 & 36,137 \\
\hline
\end{tabular}

$\mathrm{SEM}=$ tarea de asociación semántica, FON = tarea fonológica, FV = tarea de fluidez verbal, EE = error estándar. 
Tabla 4. Activación cerebro-cerebelar cruzada y patrón de activación de lateralidad de lenguaje según patrón de activación de las tareas de lenguaje

\begin{tabular}{|lcccccc|}
\hline Pacientes No ID & $\begin{array}{c}\text { Tareas RMf que detectan activación CCC } \\
\text { FV }\end{array}$ & SEM & $\begin{array}{c}\text { Actividad } \\
\text { cerebral }\end{array}$ & $\begin{array}{c}\text { Actividad } \\
\text { cerebelar }\end{array}$ & $\begin{array}{c}\text { Lateralidad } \\
\text { lenguaje }\end{array}$ \\
\hline $6,12,23,28,47,56,57$ & $\mathrm{x}$ & $\mathrm{x}$ & $\mathrm{x}$ & Izquierdo & Derecho & Típica \\
\hline 10,16 & $\mathrm{x}$ & $\mathrm{x}$ & & Izquierdo & Derecho & Típica \\
\hline 27 & $\mathrm{x}$ & $\mathrm{x}$ & Izquierdo & Derecho & Típica \\
\hline 14 & & $\mathrm{x}$ & $\mathrm{x}$ & Izquierdo & Simétrico & Típica \\
\hline 11 & $\mathrm{x}$ & $\mathrm{x}$ & Izquierdo & Izquierdo & Típica \\
\hline 48,50 & $\mathrm{x}$ & $\mathrm{x}$ & Simétrico & Derecho & Atípica \\
\hline $34,40,58$ & $\mathrm{x}$ & $\mathrm{x}$ & Simétrico & Derecho & Atípica \\
\hline 38 & $\mathrm{x}$ & $\mathrm{x}$ & $\mathrm{x}$ & Derecho & Izquierdo & Atípica \\
\hline 22 & & $\mathrm{x}$ & & Simétrico & No activación & Atípica \\
21 & & Simétrico & Derecho & Atípica \\
\hline
\end{tabular}

CCC = cerebro-cerebelar cruzado, FV = Fluidez verbal, SEM = Tarea semántica, FON = Tarea fonológica. ID = número identificador del paciente en el estudio.

sospechas de tumores cerebrales. Con estas tareas se pudo explorar de forma exitosa la lateralidad cerebral del lenguaje (típica o atípica) de estos pacientes previo a su planificación quirúrgica. El propósito del estudio fue diseñar e implementar tareas alternativas de RMf de lenguaje con estímulos lingüísticos cuyo rendimiento no se viera afectado por variables demográficas tales como edad, sexo y escolaridad, y así poder evaluar adecuadamente personas con eventuales alteraciones lingüísticas secundarias al tumor cerebral.

La tarea semántica es la tarea que tiene mejor rendimiento con menor tiempo de reacción en comparación con las otras dos tareas. Obtener registros de los tiempos de respuestas en voluntarios sanos facilitó la implementación de las secuencias de RMf para los pacientes, obteniendo así certeza del tiempo en el cual ocurre el fenómeno cognitivo-lingüístico, aspecto relevante al diseñar un paradigma de $\mathrm{RMf}^{21}$. Esto es de especial interés en pacientes con lesiones cerebrales, en los cuales sus tiempos de reacción son mayores y la modelación de la actividad cerebral en base al fenómeno cognitivo puede verse alterado por la presencia de la lesión ${ }^{3,22}$.

La FV es una de las tareas más usadas como paradigma de RMf a nivel internacional para evaluar la lateralidad de lenguaje. Sin embargo, hay que considerar el efecto que la edad puede causar en la interpretación de la actividad cerebral, pues a mayor edad, aumenta la actividad frontal, dado al esfuerzo cognitivo9. Además, esta tarea es compleja de poder realizar en pacientes que presentan alteraciones del lenguaje secundario a la presencia del tumor cerebral. Otra desventaja de la tarea de FV es que su rendimiento no puede ser controlado dentro del resonador, es por ello que su implementación para determinar la lateralidad del lenguaje debe ser interpretada en base al rendimiento cognitivo-lingüístico del paciente. Adicionalmente, es necesario que el diseño de la tarea garantice un rendimiento adecuado, en especial en pacientes con alteraciones neurológicas o en la población pediátrica ${ }^{8}$. Por lo tanto, en este estudio se optó por diseñar tareas auditivas en vez de visuales, para evitar la conversión ortográfica al código fonológico, considerando que este proceso pudiese ser complejo para pacientes con alteraciones del lenguaje.

La variabilidad de los resultados de MRf en la lateralidad del lenguaje no solo depende de los umbrales estadísticos aplicados a las imágenes MRf, sino también de los paradigmas utilizados para provocar activación lingüística ${ }^{23,24}$. Es por ello que este estudio implementa la combinación de 3 tareas de lenguaje, las cuales, en conjunto, fueron capaces de determinar la lateralidad típica y atípica del 95\% de los pacientes enrolados en este piloto. 


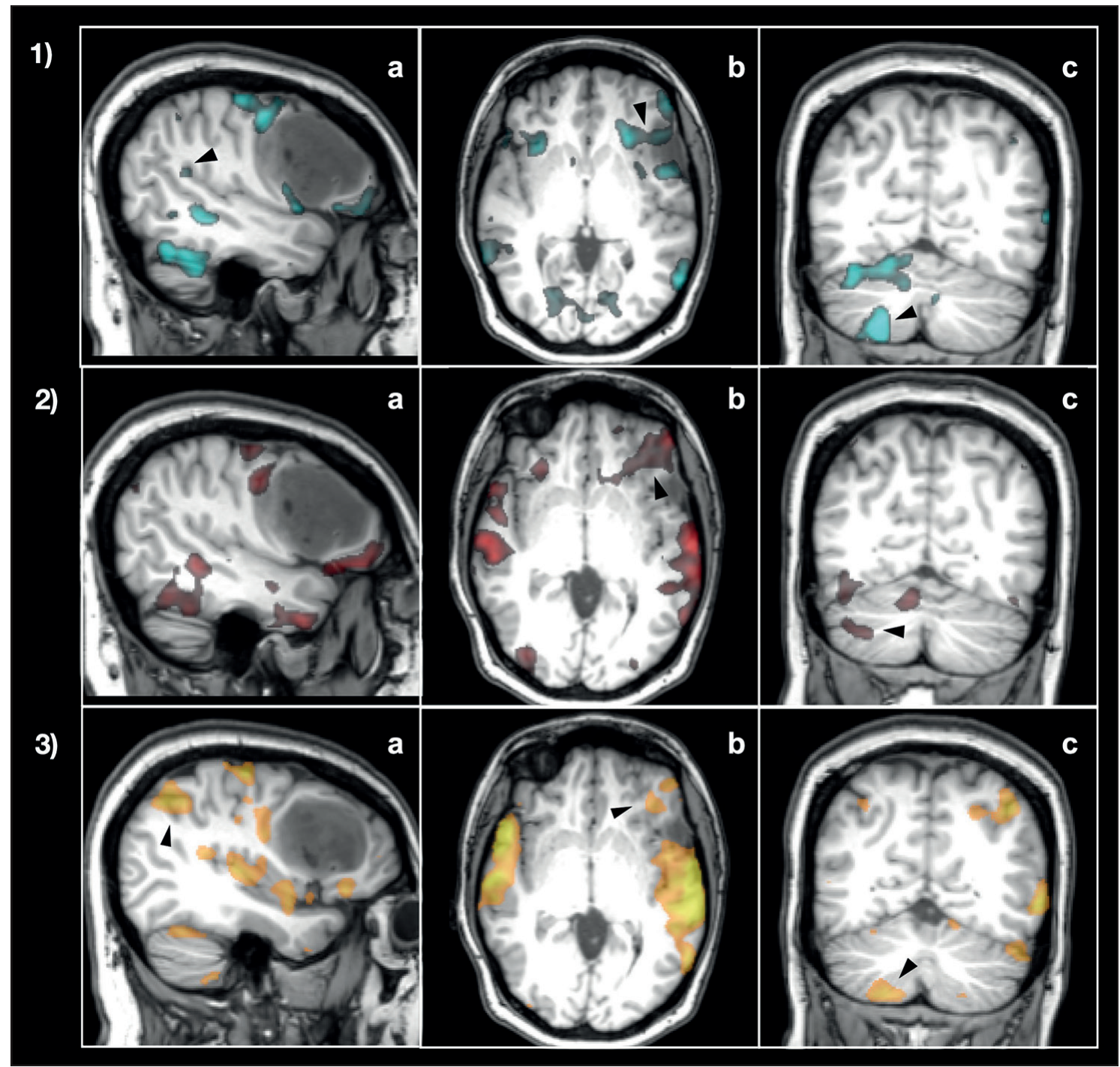

Figura 1. 1) Tarea de fluidez verbal; 2) tarea semántica; 3) tarea fonológica. Estudio de paciente portador de tumor frontal inferior izquierdo que muestra patrón de activación típico para áreas de procesamiento de lenguaje. Se observa activación supramarginal en tareas FV, FON (columna a), activación frontal inferior izquierda rodeando la lesión (columna b) y activación cerebelosa cruzada en las tres tareas (columna c).

El patrón de activación CCC se encontró presente en las otras tareas, SEM y FON, logrando replicar los resultados encontrados con la tarea de FV en estudios previos ${ }^{5,6}$. A pesar que la concordancia entre las 3 tareas es alta, se observó una mayor concordancia entre las tareas SEM y FON, siendo estas más fáciles de implementar en pacientes con alteraciones de lenguaje, dado a que su rendimiento dentro del resonador puede ser controlado.
Contar con más de un paradigma de lenguaje es crucial para determinar la lateralidad del lenguaje mediante inspección visual de patrones de activación, en especial para neuroradiólogos en entrenamiento ${ }^{13}$.

A pesar que RMf ayuda a diferenciar las regiones críticas para el procesamiento del lenguaje de aquellas regiones que apoyan este procesamiento, no pueden siempre explicar el efecto de neuro- 
plasticidad que juegan aquellas áreas no típicas para el procesamiento del lenguaje ${ }^{25}$. Si bien la RMf no es lo suficientemente precisa como para reemplazar la estimulación eléctrica cortical intraoperatoria, sí es útil para evaluar el riesgo de eventuales secuelas postoperatorias y proporciona una orientación espacial de las zonas elocuentes de lenguaje en relación a la lesión tumoral ${ }^{26}$. Además, las imágenes de RMf incorporadas a tecnología de neuronavegación intraoperatoria permiten realizar una planificación quirúrgica adecuada y un mapeo cortical personalizado, contribuyendo potencialmente a realizar resecciones tumorales más completas y seguras ${ }^{27}$. Futuros estudios deben apuntar a explorar la concordancia espacial de los patrones de actividad cortical observados con las tareas de RMf detalladas en este estudio con las zonas de activación de estas mismas tareas en forma intraoperatoria.

\section{Referencias}

1. Smits M, Wieberdink RG, Bakker SL, Dippel DW. Functional magnetic resonance imaging to determine hemispheric language dominance prior to carotid endarterectomy. J Neuroimaging. 2011; 21 (2): e162-5.

2. FitzGerald DB, Cosgrove GR, Ronner S, Jiang H, Buchbinder BR, Belliveau JW, Rosen BR, Benson RR. Location of language in the cortex: a comparison between functional MR imaging and electrocortical stimulation. AJNR Am J Neuroradiol. 1997; 18 (8): 1529-39.

3. Smits M. Functional Magnetic Resonance Imaging (fMRI) in Brain Tumour Patients. Eur Assoc NeuroOncology Mag 2012; 2: 123-.

4. Duffau H. Lessons from brain mapping in surgery for low-grade glioma: insights into associations between tumour and brain plasticity. Lancet Neurol 2005; 4: 476-86.

5. Jansen A, Flöel A, Van Randenborgh J, Konrad C, Rotte $\mathrm{M}$, Förster AF, et al. Crossed cerebro-cerebellar language dominance. Hum Brain Mapp 2005; 24 (3): 165-72.

6. Méndez Orellana C, Visch-Brink E, Vernooij M, Kalloe S, Satoer D, Vincent A, et al. Crossed Cerebrocerebellar Language Lateralization: An Additional Diagnostic Feature for Assessing Atypical Language Representation in Presurgical Functional MR Imaging. Am J Neuroradiol 2015; 36: 518-24.

7. Cho NS, Peck KK, Zhang Z, Holodny AI. Paradoxical Activation in the Cerebellum During Language fMRI in Patients with Brain Tumors: Possible Explanations
Based on Neurovascular Uncoupling and Functional Reorganization. Cerebellum 2018; 17: 286-93.

8. Sair HI, Yahyavi-Firouz-Abadi N, Calhoun VD, Airan RD, Agarwal S, Intrapiromkul J, et al. Presurgical brain mapping of the language network in patients with brain tumors using resting-state fMRI: Comparison with task fMRI. Hum Brain Mapp 2015; 37: 913-23.

9. Meinzer M, Flaisch T, Wilser L, Eulitz C, Rockstroh B, Conway $\mathrm{T}$, et al. Neural signatures of semantic and phonemic fluency in young and old adults. J Cogn Neurosci 2009; 21 (10): 2007-18.

10. Glisky EL, Riddle DR. Editores. Changes in Cognitive Function in Human Aging. In: Brain Aging: Models, Methods, and Mechanisms. CRC Press/Taylor \& Francis; Boca Raton FL. 2001. https://www.ncbi.nlm.nih.gov/ books/NBK3885/ (2007).

11. Zec RF, Burkett NR, Markwell SJ, Larsen DL. A cross-sectional study of the effects of age, education, and gender on the Boston Naming Test. Clin Neuropsychol 2007; 21: 587-616.

12. Rosselli M, Tappen R, Williams C, Salvatierra J, Zoller Y. Level of education and category fluency task among Spanish speaking elders: number of words, clustering, and switching strategies. Neuropsychol Dev Cogn B Aging Neuropsychol Cogn. 2009; 16 (6): 721-44.

13. Gore A, Hu R, Patel D, Braileanu M, Hampton D, Joshi $\mathrm{H}$, et al. Combined task activation display as an effective method to teach introductory fMRI users. Clin Imaging 2019; 55: 181-7.

14. Stippich C, Rapps N, Dreyhaupt J, Durst A, Kress B, Nennig E, et al. Localizing and lateralizing language in patients with brain tumors: feasibility of routine preoperative functional MR imaging in 81 consecutive patients. Radiology. 2007; 243 (3): 828-36.

15. Ramsey NF, Sommer IE, Rutten GJ, Kahn RS. Combined analysis of language tasks in fMRI improves assessment of hemispheric dominance for language functions in individual subjects. Neuroimage 2001; 13: 719-33.

16. Quiroga P, Albala C, Klaasen G. Validación de un test de tamizaje para el diagnóstico de demencia asociada a edad, en Chile. Rev Med Chile 2004; 132: 467-78.

17. Arora J, Pugh K, Westerveld M, Spencer S, Spencer DD, Todd Constable R. Language lateralization in epilepsy patients: fMRI validated with the Wada procedure. Epilepsia. 2009; 50 (10): 2225-41.

18. Smits M, Visch-Brink E, Schraa-Tam CK, Koudstaal PJ, van der Lugt A. Functional MR imaging of language processing: an overview of easy-to-implement paradig$\mathrm{ms}$ for patient care and clinical research. Radiographics 2006; 26 Suppl 1: S145-58.

19. Méndez Orellana C, Visch-Brink EG, De Jong-Hagels- 
tein M, Koudstaal P, Van del Lugt A, Smits M. Decreased Relative Contribution to Language Processing of the Right Hemisphere after Language Therapy Assessed with fMRI in Chronic Aphasia Patients. Procedia - Social and Behavioral Sciences 2012; 61: 20-1.

20. Méndez-Orellana C, Visch-Brink E, De Jong-Hagelstein M, Koudstaal P, van der Lugt A, Smits M. Decreased Relative Contribution to Language Proccesing of the Right Hemisphere after Language Therapy Assessed with fMRI in Chronic Aphasia Patients. Procedia Soc Behav Sci 2012; 61: 20-1.

21. Warbrick T, Reske M, Shah NJ. Transferring cognitive tasks between brain imaging modalities: implications for task design and results interpretation in FMRI studies. J Vis Exp 2014; 51793.

22. Smits M, Visch-Brink EG, Sandt-Koenderman ME van de, et al. Advanced Magnetic Resonance Neuroimaging of Language Function Recovery After Aphasic Stroke: A Technical Review. Arch Phys Med Rehabil 2012; 93: S4-S14.
23. Lurito JT, Dzemidzic M. Determination of cerebral hemisphere language dominance with functional magnetic resonance imaging. Neuroimaging Clin N Am 2001; 11 (2): 355-63.

24. Fernández G, Specht K, Weis S, Tendolkar I, Reuber M, Fell J, et al. Intrasubject reproducibility of presurgical language lateralization and mapping using fMRI. Neurology 2003; 60 (6): 969-75.

25. Duffau H. Brain plasticity and tumors. Adv Tech Stand Neurosurg 2008; 33: 3-33. doi: 10.1007/978-3-21172283-1_1.

26. Cochereau J, Deverdun J, Herbet G, Charroud C, Boyer A, Moritz-Gasser S, et al. Comparison between resting state fMRI networks and responsive cortical stimulations in glioma patients. Hum Brain Mapp 2016; 37: 3721-32.

27. Gerritsen JKW, Arends L, Klimek M, Dirven CMF, Vincent AJE. Impact of intraoperative stimulation mapping on high-grade glioma surgery outcome: a meta-analysis. Acta Neurochir (Wien) 2019; 161: 99-107.

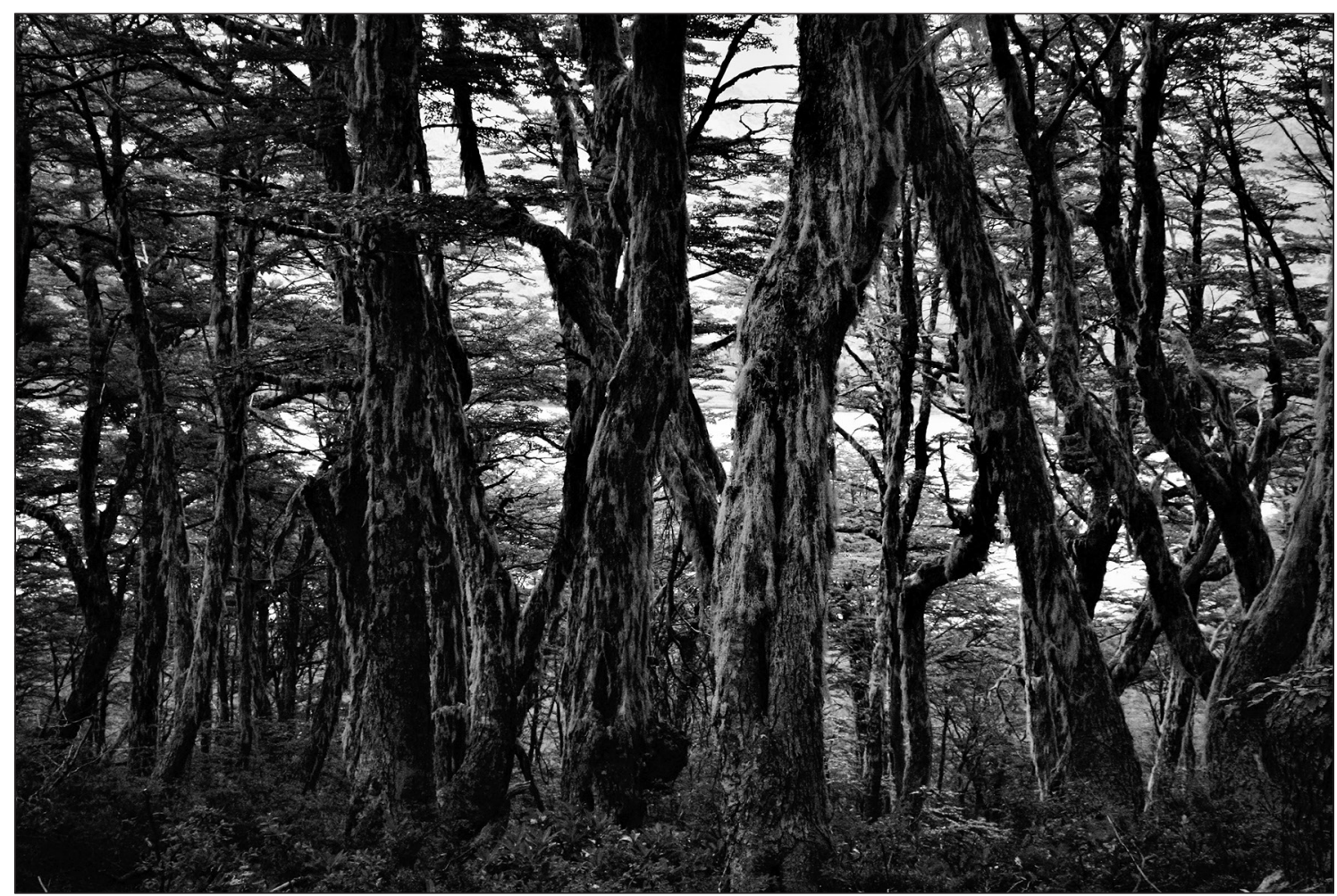

Sendero Laguna Avutardas, Curarrehue, La Araucanía. Dr. Jorge Sapunar Zenteno 